\title{
A THEORY OF FOCAL POINTS AND FOCAL INTERVALS FOR AN ELLIPTIC QUADRATIC FORM ON A HILBERT SPACE $\left({ }^{1}\right)$
}

\author{
BY \\ JOHN GREGORY
}

\begin{abstract}
The theory of focal points and conjugate points is an important part of the study of problems in the calculus of variations and control theory. Hestenes has shown that for many problems this theory may be studied by Hilbert space methods. In a previous paper the author has extended the theory of Hestenes to elliptic quadratic forms $J(x ; \sigma)$ defined on $\mathfrak{U}(\sigma)$ where $\sigma$ is a member of the metric space $(\Sigma, \rho)$ and $\mathfrak{A}(\sigma)$ denotes a closed subspace of $\mathfrak{A}$. A fundamental part of this extension is concerned with inequalities dealing with the signature $s(\sigma)$ and nullity $n(\sigma)$ of $J(x ; \sigma)$ on $\mathfrak{Q}(\sigma)$ where $\sigma$ is in a $\rho$ neighborhood of a fixed point $\sigma_{0}$ in $\Sigma$.

The purpose of this paper is threefold. The first purpose is to show that the extended theory includes the focal point hypotheses of Hestenes. The second purpose is to obtain a focal point theory much like that of Hestenes. It is interesting to note that our theory is based only on the nonnegative integers $s(\lambda)$ and $n(\lambda)$. This will facilitate later work on numerical calculations of focal points. Our final purpose is to obtain an abstract focal interval theory in which the usual focal points are (degenerate) focal intervals.

While previous authors have considered specific problems, no general results for the focal interval case seem to be contained in the literature. An expression for the number of focal intervals on a subinterval $\left(\lambda^{\prime}, \lambda^{\prime \prime}\right)$ of $[a, b]$ is given. This expression is a key result for our work on approximation of focal intervals (to be published).

For completeness we give comparison theorems for focal point problems. In addition an example is given for problems in optimal control theory. The correspondence between our focal intervals and solutions to the differential equations of the example is given.
\end{abstract}

I. Preliminaries. We now state the approximation hypothesis given in [3] and [4] and the focal point hypothesis of Hestenes given in [6]. The former is contained in (1) and (2), the latter in (3). $\mathfrak{A}$ will denote a Hilbert space with inner product $(x, y)$ and norm $\|x\|=(x, x)^{1 / 2}$. Strong convergence will be denoted by $x_{q} \Rightarrow x_{0}$ and weak convergence by $x_{q} \rightarrow x_{0}$.

Let $\Sigma$ be a metric space with metric $\rho$. A sequence $\left\{\sigma_{r}\right\}$ in $\Sigma$ converges to $\sigma_{0}$ in $\Sigma$, written $\sigma_{r} \rightarrow \sigma_{0}$, if $\lim _{r=\infty} \rho\left(\sigma_{r}, \sigma_{0}\right)=0$. For each $\sigma$ in $\Sigma$ let $\mathfrak{A}(\sigma)$ be a closed subspace of $\mathfrak{A}$ such that

Received by the editors June 29, 1970.

AMS 1969 subject classifications. Primary 4615, 49XX, 9340.

Key words and phrases. Focal points, conjugate points, calculus of variations, control theory, Hilbert space, quadratic forms.

(1) The preparation of this paper was sponsored in part by the U.S. Army Research Office under Grant DA-31-124-ARO(D)-355. Reproduction in whole or in part is permitted for any purpose of the United States Government.

Copyright (C) 1971, American Mathematical Society 
(1a) if $\sigma_{r} \rightarrow \sigma_{0}, x_{r}$ in $\mathfrak{A}\left(\sigma_{r}\right), x_{r} \rightarrow y_{0}$ then $y_{0}$ is in $\mathfrak{A}\left(\sigma_{0}\right)$, and

(1b) if $x_{0}$ is in $\mathfrak{A}\left(\sigma_{0}\right)$ and $\varepsilon>0$ there exists $\delta>0$ such that, whenever $\rho\left(\sigma, \sigma_{0}\right)<\delta$, there exists $x_{\sigma}$ in $\mathfrak{A}(\sigma)$ satisfying $\left\|x_{0}-x_{\sigma}\right\|<\varepsilon$.

For each $\sigma$ in $\Sigma$ let $J(x ; \sigma)$ be a quadratic form defined on $\mathfrak{U}(\sigma)$ with $J(x, y ; \sigma)$ the associated bilinear form. For $r=0,1,2, \ldots$ let $x_{r}$ be in $\mathfrak{A}\left(\sigma_{r}\right), y_{r}$ in $\mathfrak{A}\left(\sigma_{r}\right)$ such that if $x_{r} \rightarrow x_{0}, y_{r} \Rightarrow y_{0}$, and $\sigma_{r} \rightarrow \sigma_{0}$ then

(2a) $\lim _{r=\infty} J\left(x_{r}, y_{r} ; \sigma_{r}\right)=J\left(x_{0}, y_{0} ; \sigma_{0}\right)$,

(2b) $\lim \inf _{r=\infty} J\left(x_{r} ; \sigma_{r}\right) \geqq J\left(x_{0} ; \sigma_{0}\right)$, and

(2c) $\lim _{r=\infty} J\left(x_{r} ; \sigma_{r}\right)=J\left(x_{0} ; \sigma_{0}\right)$ implies $x_{r} \Rightarrow x_{0}$.

Let $a, b$ be real numbers $(a<b)$ and define $\Lambda=[a, b]$. Let $\{\mathfrak{H}(\lambda): \lambda$ in $\Lambda\}$ be a one parameter family of closed subspaces of $\mathfrak{A}$ such that $\mathfrak{A}(a)=0, \mathfrak{A}(b)=\mathfrak{A}$, and $\mathfrak{U}\left(\lambda_{1}\right) \subset \mathfrak{A}\left(\lambda_{2}\right)$ whenever $\lambda_{1}, \lambda_{2}$ in $\Lambda, \lambda_{1}<\lambda_{2}$. In this paper we will require that one (or both) parts of the additional hypothesis is satisfied:

(3a) $\mathfrak{A}\left(\lambda_{0}\right)=\bigcap_{\lambda_{0}<\lambda \leqq b} \mathfrak{A}(\lambda)$ whenever $a \leqq \lambda_{0}<b$, and

(3b) $\mathfrak{A}\left(\lambda_{0}\right)=\mathrm{Cl}\left(\bigcup_{a \leqq \lambda<\lambda_{0}} \mathfrak{A}(\lambda)\right)$ whenever $a<\lambda_{0} \leqq b$.

LEMMA 1. If $\mathfrak{B}$ is a closed subspace of $\mathfrak{A},\left\{x_{n}\right\} \subset \mathfrak{B}, x_{n} \rightarrow y_{0}$ then $y_{0}$ in $\mathfrak{B}$.

The proof of this lemma is straightforward and will be omitted.

In this paper we will consider (1) and (2) in the " $\lambda$-setting". Thus we set $\Sigma=\Lambda$ $=[a, b]$ and $\rho\left(\lambda_{1}, \lambda_{2}\right)=\left|\lambda_{2}-\lambda_{1}\right|$. We now show that the hypotheses in (3) are stronger than those in (1). The converse of Theorem 2 holds in our setting and is left as an exercise.

THEOREM 2. Hypothesis (3) implies (1). In particular (3a) implies (1a) while (3b) implies (1b).

For (1a) let $\lambda_{r} \rightarrow \lambda_{0}, x_{r}$ in $\mathfrak{A}\left(\lambda_{r}\right)$ and $x_{r} \rightarrow y_{0}$. If there exists a subsequence $\left\{\lambda_{r_{k}}\right\}$ such that $\lambda_{r_{k}} \nearrow \lambda_{0}$ we have $x_{r_{k}} \subset \mathfrak{A}\left(\lambda_{0}\right)$ so that $y_{0}$ is in $\mathfrak{A}\left(\lambda_{0}\right)$ by Lemma 1 .

Thus assume $\lambda_{r} \searrow \lambda_{0}$ and $\lambda_{r} \neq b$. Let $\bar{\lambda}$ be given and satisfy $\lambda_{0}<\bar{\lambda} \leqq b$. By (3a) there exists $N$ such that $r \geqq N$ implies $x_{r}$ in $\mathfrak{A}(\bar{\lambda})$. By Lemma $1, y_{0}$ is in $\mathfrak{A}(\bar{\lambda})$. Finally $y_{0}$ in $\mathfrak{A}\left(\lambda_{0}\right)$ follows from (3a).

For (1b) assume $x_{0}$ is $\mathfrak{A}\left(\lambda_{0}\right)$ and $\varepsilon>0$ are given. We assume $\lambda_{0} \neq a$; if $\lambda_{0}=a$ the result follows immediately as $x_{0}=0$. From (3b) there exists $\bar{\lambda}\left(a \leqq \bar{\lambda}<\lambda_{0}\right), \bar{x}$ in $\mathfrak{A}(\bar{\lambda})$, such that $\left\|x-x_{0}\right\|<\varepsilon$. Let $\delta \leqq \lambda_{0}-\bar{\lambda}$. Then $\lambda \geqq \lambda_{0}-\delta$ implies $\bar{\lambda} \leqq \lambda_{0}-\delta<\lambda$, $\mathfrak{U}(\bar{\lambda}) \subset \mathfrak{U}(\lambda)$ and hence $\bar{x}$ in $\mathfrak{A}(\lambda)$. This completes the proof.

The form $J(x)$ is elliptic on $\mathfrak{A}$ if conditions (2b) and (2c) hold with $J(x)$ replacing $J(x ; \sigma)$ and $\mathfrak{A}$ replacing $\mathfrak{A}(\sigma)$. Let $J(x ; \lambda)$ denote the restriction of $J(x)$ to $\mathfrak{A}(\lambda)$. The following theorem is immediate as $J(x)$ is elliptic on $\mathfrak{A}$.

THEOREM 3. The forms $J(x ; \lambda)$ satisfy hypothesis $(2)$.

The signature and nullity of $J(x)$ restricted to $\mathfrak{A}(\lambda)$ are now defined. The signature (index) of $J(x ; \lambda)$, written $s(\lambda)$, is the dimension of a maximal linear subclass $\mathfrak{B}$ of $\mathfrak{A}(\lambda)$ on which $J(x ; \lambda)<0$ for $x \neq 0$. The nullity of $J(x ; \lambda)$, written $n(\lambda)$, on $\mathfrak{A}(\lambda)$ is the dimension of the subspace $\mathscr{C}$ of $\mathfrak{A}(\lambda)$ where $\mathscr{C}=\{y$ in $\mathfrak{A}(\lambda) \mid J(x, y ; \lambda)=0$ for 
all $x$ in $\mathfrak{A}(\lambda)\}$. We will denote the set $\mathscr{C}$ by $\mathfrak{A}_{0}(\lambda)$. The symbolism $s(\lambda-0)$ is used to denote the left-hand limit of $s(\lambda)$. Similar remarks hold for $s(\lambda+0), n(\lambda-0)$ and $n(\lambda+0)$. We set $s(a-0)=0, n(a-0)=0$, and $s(b+0)=s(b)+n(b)$.

Theorem 4 has been given in [6].

THEOREM 4. The quantity $s(\lambda)$ is the dimension of a maximal subspace $\mathscr{E}$ of $\mathfrak{U}(\lambda)$ on which $J(x ; \lambda) \leqq 0$ such that $\mathscr{E} \cap \mathfrak{U}_{0}(\lambda)=0$. The sum $m(\lambda)=s(\lambda)+n(\lambda)$ is given by the quantity: The dimension of a maximal subspace $\mathscr{D}$ of $\mathfrak{A}(\lambda)$ in which $J(x ; \lambda) \leqq 0$. Thus the quantities $s(\lambda)$ and $m(\lambda)$ are nondecreasing functions on $\Lambda$.

A point $\lambda$ at which $s(\lambda)$ is discontinuous will be called a focal point of $J(x)$ relative to $\mathfrak{A}(\lambda)(\lambda$ in $\Lambda)$. The difference $f(\lambda)=s(\lambda+0)-s(\lambda-0)$ will be called the order of $\lambda$ as a focal point. A focal point $\lambda$ will be counted the number of times equal to its order.

II. Inequalities. We now give inequalities involving $s(\lambda)$ and $n(\lambda)$. We note that inequalities (4), (5), and (6) have been given in the more general $\sigma$-setting of hypotheses (1) and (2). Thus they follow immediately by Theorems 2 and 3 .

THEOREM 5. Assume hypothesis (3a) holds. Let $\lambda_{0}$ in $\Lambda$ be given. Then there exists $\delta>0$ such that $\lambda$ in $\Lambda$ and $\left|\lambda-\lambda_{0}\right|<\delta$ imply

$$
s(\lambda)+n(\lambda) \leqq s\left(\lambda_{0}\right)+n\left(\lambda_{0}\right) .
$$

In particular $s(a+0)=n(a+0)=0$. Finally $s\left(\lambda_{0}\right)+n\left(\lambda_{0}\right)=s\left(\lambda_{0}+0\right)+n\left(\lambda_{0}+0\right)$.

Inequality (4) holds by our discussion. The second result follows as $s(a)+n(a)=0$. The final result holds by Theorem 4 .

THEOREM 6. Assume hypothesis (3b) holds. Let $\lambda_{0}$ in $\Lambda$ be given. Then there exists $\delta>0$ such that $\lambda$ in $\Lambda$ and $\left|\lambda-\lambda_{0}\right|<\delta$ imply

$$
s\left(\lambda_{0}\right) \leqq s(\lambda) \text {. }
$$

In particular $s\left(\lambda_{0}-0\right)=s\left(\lambda_{0}\right)$.

Inequality (5) holds by our discussion. The remaining result holds by Theorem 4.

THEOREM 7. Assume hypothesis (3) holds. Let $\lambda_{0}$ in $\Lambda$ be given. Then there exists $\delta>0$ such that $\lambda$ in $\Lambda$ and $\left|\lambda-\lambda_{0}\right|<\delta$ imply

$$
s\left(\lambda_{0}\right) \leqq s(\lambda) \leqq s(\lambda)+n(\lambda) \leqq s\left(\lambda_{0}\right)+n\left(\lambda_{0}\right) .
$$

In addition we have, for such $\lambda$,

(7) $n\left(\lambda_{0}\right)=0$ implies $s(\lambda)=s\left(\lambda_{0}\right)$ and $n(\lambda)=0$,

(8a) $n(\lambda) \leqq n\left(\lambda_{0}\right)$, and

(8b) $n(\lambda)=n\left(\lambda_{0}\right)$ implies $s(\lambda)=s\left(\lambda_{0}\right)$ and $m(\lambda)=m\left(\lambda_{0}\right)$.

THEOREM 8. Assume $\lambda_{1} \neq \lambda_{2}$ in $\Lambda$ implies $\mathfrak{A}_{0}\left(\lambda_{1}\right) \cap \mathfrak{A}_{0}\left(\lambda_{2}\right)=0$. Then $a \leqq \lambda<\lambda_{1} \leqq b$ implies $s(\lambda)+n(\lambda) \leqq s\left(\lambda_{1}\right)$. In addition if (3a) holds then $s(\lambda+0)=s(\lambda)+n(\lambda)$. If (3) holds then $f(\lambda)=n(\lambda)$ and the set $\Lambda_{1}=\{\lambda$ in $\Lambda \mid n(\lambda) \neq 0\}$ is finite. 
For the first result let $\mathscr{C}=\mathscr{B} \oplus \mathfrak{A}_{0}(\lambda)$ be a subspace of $\mathfrak{A}(\lambda)$ where $\mathfrak{B}$ is a maximal space such that $x \neq 0$ in $\mathfrak{B}$ implies $J(x ; \lambda)<0$. By Theorem 4 we have $s(\lambda)+n(\lambda)$ $\leqq s\left(\lambda_{1}\right)$. If (3a) holds we have the inequalities

$$
s(\lambda+0) \leqq s(\lambda+0)+n(\lambda+0)=n(\lambda)+s(\lambda) \leqq s(\lambda+0) .
$$

Finally if (3b) also holds we have $f(\lambda)=s(\lambda+0)-s(\lambda)+s(\lambda)-s(\lambda-0)=n(\lambda)$. Thus $\Lambda_{1}$ is finite as $s(b)$ is.

III. Focal point theory. We note that Theorem 9 characterizes the "usual" focal point problem. In Theorems 10 to 13 we allow a nonzero vector $x$ to belong to $\mathfrak{A}_{0}\left(\lambda_{1}\right) \cap \mathfrak{A}_{0}\left(\lambda_{2}\right)$. This gives rise to a focal interval theory. For convenience we will assume that (3) holds for the remainder of this section. The cases where (3a) or (3b) hold separately will follow immediately with weaker results.

The following theorem follows immediately. It characterizes the usual focal point problem.

THEOREM 9. If (3) holds and if $\mathfrak{A}_{0}\left(\lambda_{1}\right) \cap \mathfrak{A}_{0}\left(\lambda_{2}\right)=0$ when $\lambda_{1} \neq \lambda_{2}$, then $f(a)=0$ and $f(\lambda)=n(\lambda)$ on $a \leqq \lambda \leqq b$. Thus if $\lambda_{0}$ in $\Lambda$ the following quantities are equal:

(9a) the number of focal points on $a \leqq \lambda<\lambda_{0}$,

(9b) the signature $s\left(\lambda_{0}\right)$ of $J(x)$ on $\mathfrak{A}\left(\lambda_{0}\right)$,

(9c) the sum $\sum_{a \leqq \lambda<\lambda_{0}} n(\lambda)$, and

(9d) the sum $\sum\left[s\left(\lambda_{i}+0\right)-s\left(\lambda_{i}\right)\right]$ taken over all $\lambda_{i}$ such that $a \leqq \lambda_{i}<\lambda_{0}$ and $s(\lambda)$ discontinuous at $\lambda_{i}$.

We will denote the set $\{x \mid J(x, y)=0$ for all $y$ in $\mathfrak{A}(\lambda)\}$ by $\mathfrak{A}(\lambda)^{J}$. Clearly $\mathfrak{A}_{0}(\lambda)$ $=\mathfrak{A}(\lambda) \cap \mathfrak{A}(\lambda)^{J}$.

LEMMA 10. If $\lambda_{1}<\lambda_{2}$ and $x \neq 0$ in $\mathfrak{A}_{0}\left(\lambda_{1}\right) \cap \mathfrak{A}_{0}\left(\lambda_{2}\right)$ then $x$ in $\mathfrak{A}_{0}(\lambda)$ for $\lambda_{1} \leqq \lambda \leqq \lambda_{2}$.

For $\bar{\lambda}$ in $\lambda_{1} \leqq \lambda \leqq \lambda_{2}$ we have $\mathfrak{A}\left(\lambda_{1}\right) \subset \mathfrak{U}(\bar{\lambda})$ and $\mathfrak{U}\left(\lambda_{2}\right)^{J} \subset \mathfrak{U}(\bar{\lambda})^{J}$. Thus $\mathfrak{A}_{0}\left(\lambda_{1}\right) \cap \mathfrak{A}_{0}\left(\lambda_{2}\right)$ $=\mathfrak{A}\left(\lambda_{1}\right) \cap \mathfrak{H}\left(\lambda_{2}\right)^{J} \subset \mathfrak{A}(\bar{\lambda}) \cap \mathfrak{A}(\bar{\lambda})^{J}=\mathfrak{A}_{0}(\bar{\lambda})^{J}$.

LEMMA 11. If $x \neq 0$ in $\mathfrak{A}_{0}(\lambda), \lambda_{1}<\lambda<\lambda_{2}$, then $x$ is in $\mathfrak{A}_{0}(\lambda), \lambda_{1} \leqq \lambda \leqq \lambda_{2}$.

Clearly $x$ is in $\mathfrak{A}\left(\lambda_{2}\right)$. If $x$ is not in $\mathfrak{A}\left(\lambda_{2}\right)^{J}$ there exists $y$ in $\mathfrak{A}\left(\lambda_{2}\right)$ such that $J(x, y)$ $=1$. We may choose sequences $\left\{\mu_{n}\right\}$ and $\left\{y_{n}\right\}$ such that $y_{n}$ in $\mathfrak{A}\left(\mu_{n}\right), y_{n} \Rightarrow y$ as $\mu_{n} \nearrow \lambda_{2}$. Thus $1=J(x, y)=\lim _{n=\infty} J\left(x, y_{n}\right)=0$. Thus $x$ is in $\mathfrak{A}_{0}\left(\lambda_{2}\right)$.

Conversely if $\bar{\lambda}$ satisfies $\lambda_{1}<\bar{\lambda}<\lambda_{2}$ then $x$ is in $\mathfrak{A}(\bar{\lambda})^{J} \subset \mathfrak{A}\left(\lambda_{1}\right)^{J}$. But $x$ is in $\bigcap_{\lambda_{1}<\lambda} \mathfrak{A}(\lambda)=\mathfrak{A}\left(\lambda_{1}\right)$. Thus $x$ is in $\mathfrak{A}_{0}\left(\lambda_{1}\right)$.

THEOREM 12. If $x \neq 0$ in $\mathfrak{A}_{0}\left(\lambda_{1}\right) \cap \mathfrak{A}_{0}\left(\lambda_{2}\right), \lambda_{1} \neq \lambda_{2}$, then there exists a closed interval $\Lambda_{1}=\left\{\lambda \mid \lambda^{\prime} \leqq \lambda \leqq \lambda^{\prime \prime}\right\}$ of $\Lambda$ such that $\left\{\lambda \mid x\right.$ in $\left.\mathfrak{2}_{0}(\lambda)\right\}=\Lambda_{1}$.

Let $\lambda^{\prime}=\operatorname{glb}\left\{\lambda \mid x\right.$ in $\left.\mathfrak{A}_{0}(\lambda)\right\}$ and $\lambda^{\prime \prime}=\operatorname{lub}\left\{\lambda \mid x\right.$ in $\left.\mathfrak{A}_{0}(\lambda)\right\}$. By Lemma $10, x$ is in $\mathfrak{A}_{0}(\lambda), \lambda^{\prime}<\lambda<\lambda^{\prime \prime}$. The theorem now follows by Lemma 11 .

Theorem 13 restates inequalities involving the indices $s(\lambda)$ and $n(\lambda)$ which characterizes focal interval problems. 
THEOREM 13. Let $\lambda_{0}$ be given such that $a<\lambda_{0}<b$. Then the following inequalities hold.

(10a) $s\left(\lambda_{0}-0\right)=s\left(\lambda_{0}\right), s\left(\lambda_{0}+0\right) \geqq s\left(\lambda_{0}\right)$,

(10b) $n\left(\lambda_{0}\right) \geqq n\left(\lambda_{0}-0\right), n\left(\lambda_{0}\right) \geqq n\left(\lambda_{0}+0\right)$,

(10c) $s\left(\lambda_{0}+0\right)-s\left(\lambda_{0}\right)=n\left(\lambda_{0}\right)-n\left(\lambda_{0}+0\right) \geqq 0$.

All results have been given above.

It is instructive to "describe" two types of focal point phenomena. For convenience we will describe the first type as "focal point theory" and the second type as "focal interval theory." In the former, a vector $x \neq 0$ cannot be a $J$ null vector of two distinct spaces $\mathfrak{A}\left(\lambda_{1}\right)$ and $\mathfrak{A}\left(\lambda_{2}\right), \lambda_{1} \neq \lambda_{2}$. In the latter case a vector $x \neq 0$ is a $J$ null vector of $\mathfrak{A}(\lambda)\left(\lambda\right.$ in $\left.\Lambda_{1}\right)$ where $\Lambda_{1}$ is a closed subinterval of $\Lambda$. We allow $\Lambda$ to be a singleton. Thus focal points are focal intervals.

Focal Point Theory is characterized by Theorem 9. A graph of $\lambda$ versus $s(\lambda)$ shows that the discontinuities in $s(\lambda)$ are at points at which $n(\lambda) \neq 0$; in fact the value of the jump at $\lambda$ is $n(\lambda)$.

Focal Interval Theory is characterized by Theorems 12 and 13. A graph of $\lambda$ versus $s(\lambda)$ and $n(\lambda)$ shows that the points of interest are those in which $n(\lambda)$ is discontinuous. If the nullity is increasing at $\lambda, n(\lambda)$ is continuous from the right. If the nullity is decreasing at $\lambda, n(\lambda)$ is continuous from the left and the signature increases by an amount equal to $n(\lambda)-n(\lambda+0)$.

We continue our study of focal interval theory by defining "focal intervals". For this purpose we assume $\lambda_{1}<\lambda_{2}<\cdots<\lambda_{p}$ are the distinct focal points on $\Lambda$ where $e_{i}$ will denote the order of $\lambda_{i}$ as a focal point. For convenience the space $\mathfrak{A}_{0}(\lambda)$ will be called the $J$ null vectors of $\mathfrak{A}(\lambda)$. Let $E_{\mathfrak{i}}$ denote the $J$ null vectors of $\mathfrak{A}\left(\lambda_{i}\right)$ which are not $J$ null vectors of $\mathfrak{A}(\lambda), \lambda>\lambda_{i}$. The following procedure is given to make "focal intervals" well defined.

To obtain the $e_{1}$ focal intervals $I_{1}, \ldots, I_{e_{1}}$ associated with (which end at) $\lambda_{1}$ we proceed as follows: choose $I_{1}=\left[\xi_{11}, \lambda_{1}\right]$ where $\xi_{11}=\min \left\{\lambda \leqq \lambda_{1} \mid x\right.$ in $\mathfrak{A}_{0}(\lambda)$ for some $x \neq 0$ in $\left.E_{1}\right\}$. Let $x_{11}$ be the vector giving $\xi_{11}$. Choose $I_{2}=\left[\xi_{21}, \lambda_{1}\right]$ where $\xi_{21}=\min \left\{\lambda \leqq \lambda_{1} \mid x\right.$ in $\mathfrak{A}_{0}(\lambda)$ for some $x \neq 0$ in $E_{1}$ and $\left.\left(x, x_{11}\right)=0\right\}$. Let $x_{21}$ be the vector giving $\xi_{21} . \cdots$ Choose $I_{e_{1}}=\left[\xi_{e_{1} 1}, \lambda_{1}\right]$ where $\xi_{e_{1} 1}=\min \left\{\lambda \leqq \lambda_{1} \mid x\right.$ in $\mathfrak{A}_{0}(\lambda)$ for some $x \neq 0$ in $E_{1}$ and $\left.\left(x, x_{k 1}\right)=0 ; k=1, \ldots, e_{1}-1\right\}$. If $x_{e_{1} 1}$ is the vector giving $\xi_{e_{1} 1}$ then $\mathfrak{A}_{0}\left(\lambda_{1}\right)$ is the direct sum of the span of $\left\{x_{11}, x_{21}, \ldots, x_{e_{1} 1}\right\}$ and $\mathfrak{A}_{0}\left(\lambda_{1}\right) \cap \mathfrak{A}_{0}\left(\lambda_{1}+0\right)$.

With obvious modifications we define the $e_{2}$ focal intervals $I_{e_{1}+1}, \ldots, I_{e_{1}+e_{2}}$ associated with $\lambda_{2} ; \ldots$; the $e_{p}$ focal intervals $I_{k}, \ldots, I_{l}$ associated with $\lambda_{p}\left(k=e_{1}\right.$ $\left.+e_{2}+\cdots+e_{p-1}+1 ; l=e_{1}+e_{2}+\cdots+e_{p}\right)$.

Let $\lambda^{\prime}, \lambda^{\prime \prime}$ in $\Lambda$ with $a \leqq \lambda^{\prime}<\lambda^{\prime \prime} \leqq b$. We denote the number of focal intervals on $\left(\lambda^{\prime}, \lambda^{\prime \prime}\right)$ by $f\left(\lambda^{\prime}, \lambda^{\prime \prime}\right)$, the number of focal intervals on $\left(a, \lambda^{\prime \prime}\right)$ containing the point $\lambda^{\prime}$ by $g\left(\lambda^{\prime}, \lambda^{\prime \prime}\right)$, and the dimension of the space $\mathfrak{A}_{0}\left(\lambda^{\prime}\right) \ominus \mathfrak{A}_{0}\left(\lambda^{\prime}\right) \cap \mathfrak{A}_{0}\left(\lambda^{\prime \prime}\right)$ by $r n\left(\lambda^{\prime}, \lambda^{\prime \prime}\right)$.

LEMMA 14. If $\lambda^{\prime}, \lambda^{\prime \prime}$ in $\Lambda, a \leqq \lambda^{\prime}<\lambda^{\prime \prime}<b$, then $f\left(a, \lambda^{\prime \prime}\right)=f\left(a, \lambda^{\prime}\right)+f\left(\lambda^{\prime}, \lambda^{\prime \prime}\right)+g\left(\lambda^{\prime}, \lambda^{\prime \prime}\right)$. 
$I$ is a focal interval on $\left(a, \lambda^{\prime \prime}\right)$ if and only if it satisfies one of the following three mutual exclusive descriptions: $I$ is a focal interval on $\left(a, \lambda^{\prime}\right), I$ is a focal interval on $\left(\lambda^{\prime}, \lambda^{\prime \prime}\right)$, or $I$ is a focal interval on $\left(a, \lambda^{\prime \prime}\right)$ containing $\lambda^{\prime}$.

LEMma 15. If $\lambda$ in $\Lambda$ then $f(a, \lambda)=s(\lambda)$.

The result follows by the definition of $I_{k}$.

Lemma 16. If $a \leqq \lambda^{\prime}<\lambda^{\prime \prime}<b$ then $g\left(\lambda^{\prime}, \lambda^{\prime \prime}\right)=r n\left(\lambda^{\prime}, \lambda^{\prime \prime}\right)$.

The number of focal intervals on $\left(a, \lambda^{\prime \prime}\right)$ containing $\lambda^{\prime}$ is equal to the number of null vectors of $\mathfrak{A}\left(\lambda^{\prime}\right)$ not contained in $\mathfrak{A}\left(\lambda^{\prime \prime}\right)$.

The next result now follows immediately.

THEOREM 17. If $\lambda^{\prime}, \lambda^{\prime \prime}$ in $\Lambda, a \leqq \lambda^{\prime \prime}<b$, then

$$
f\left(\lambda^{\prime}, \lambda^{\prime \prime}\right)=s\left(\lambda^{\prime \prime}\right)-s\left(\lambda^{\prime}\right)-r n\left(\lambda^{\prime}, \lambda^{\prime \prime}\right) .
$$

We note for completeness that Hestenes, in unpublished notes, has proven the following result: If $\mathscr{B}$ is a closed subspace of $\mathfrak{A}$ then $s(\mathfrak{U})=s(\mathscr{B})+s(\mathscr{B})+r n(\mathscr{B})$ where $\mathscr{B}^{J}$ are the $J$ orthogonal vectors of $\mathscr{B}, r n(\mathscr{B})$ is the dimension of $\mathscr{B}_{0} \ominus \mathscr{B}_{0} \cap \mathfrak{A}_{0}$. Thus the number of focal points on $\left(\lambda^{\prime}, \lambda^{\prime \prime}\right)$ is equal to $s\left(\mathscr{B}^{J}\right)$.

Let $m\left(\lambda^{\prime}, \lambda^{\prime \prime}\right)$ denote the dimension of the space $\mathfrak{A}_{0}\left(\lambda^{\prime}\right) \cap \mathfrak{A}_{0}\left(\lambda^{\prime \prime}\right)$. Noting that $n\left(\lambda^{\prime}\right)=r n\left(\lambda^{\prime}, \lambda^{\prime \prime}\right)+m\left(\lambda^{\prime}, \lambda^{\prime \prime}\right)$ we have

Corollary 18. If $\lambda^{\prime}, \lambda^{\prime \prime}$ in $\Lambda, a \leqq \lambda^{\prime}<\lambda^{\prime \prime}<b$, then

$$
f\left(\lambda^{\prime}, \lambda^{\prime \prime}\right)=s\left(\lambda^{\prime \prime}\right)-\left[s\left(\lambda^{\prime}\right)+n\left(\lambda^{\prime}\right)\right]+m\left(\lambda^{\prime}, \lambda^{\prime \prime}\right) .
$$

IV. Comparison theorems. We now consider comparison theorems for focal point problems. These results have been given by Hestenes [6] and Lopez [8] and are included for completeness. We note that these results depend only on the discontinuity of $s(\lambda)$ and not upon what the discontinuity is due to. Thus we need not assume that either (3a) or (3b) hold.

THEOREM 19. Let $\mathfrak{A}^{*}$ be a subspace of $\mathfrak{A}, \mathfrak{A} *(\lambda)=\mathfrak{A}(\lambda) \cap \mathfrak{A}^{*}$ for $\lambda$ in $\Lambda$. Let $\lambda_{1} \leqq \lambda_{2} \leqq \cdots \leqq \lambda_{m}$ be the focal points of $J(x)$ relative to $\mathfrak{A}(\lambda), \lambda$ in $\Lambda$ and let $\lambda_{1} \leqq \lambda_{2} \leqq \cdots \leqq \lambda_{m^{*}}^{*}$ be the focal points of $J(x)$ relative to $\mathfrak{H}^{*}(\lambda), \lambda$ in $\Lambda$. Then the relations

$$
\lambda_{r} \leqq \lambda_{r}^{*} \quad\left(r=1, \ldots, m^{*}\right)
$$

hold. If $\mathfrak{A}^{*}=\mathfrak{A} \ominus \mathscr{D}, d=\operatorname{dim} \mathscr{D}$ then we have in addition

$$
\lambda_{r}^{*} \leqq \lambda_{r+d}
$$

provided that $r+d \leqq m$.

THEOREM 20. Let $J^{*}(x)$ be a second quadratic form on $\mathfrak{A}$ such that $J^{*}(x) \geqq J(x)$ on $\mathfrak{A}$. Let $\lambda_{1} \leqq \lambda_{2} \leqq \cdots \leqq \lambda_{m}$ and $\lambda_{1}^{*} \leqq \lambda_{2}^{*} \leqq \cdots \leqq \lambda_{m^{*}}^{*}$ be respectively the focal points of $J(x)$ and $J^{*}(x)$ relative to $\mathfrak{A}(\lambda), \lambda$ in $\Lambda$. Then the inequalities

$$
\lambda_{r} \leqq \lambda_{r}^{*} \quad\left(r=1, \ldots, m^{*}\right)
$$


hold. If (6.1d) holds and $J^{*}(x)>J(x)$ for all $x \neq 0$ in $\mathfrak{A}$ then

$$
\lambda_{r}<\lambda_{r}^{*} \quad\left(r=1, \ldots, m^{*}\right) .
$$

V. An example. We now consider an example to simultaneously illustrate the usual focal point phenomena and focal interval phenomena. The setting will be in the theory of optimal control problems. Many details for this example are found in [9].

An element $x$ of $\mathfrak{A}$ is an arc

$$
x: x^{i}(t), \quad u^{k}(t) \quad(a \leqq t \leqq b) \quad(i=1, \ldots, n ; k=1, \ldots, q)
$$

where $x^{i}(t)$ and $u^{k}(t)$ are Lebesgue square integrable functions. The subspace $\mathfrak{B}$ of $\mathfrak{A}$ will denote all arcs which also satisfy

$$
\dot{x}=A x+B u \quad \text { and } \quad C^{*} x(a)=0 .
$$

Finally $\mathscr{C}$ will denote all arcs $x$ in $\mathscr{B}$ which also satisfy $x(b)=0$.

The quadratic form

$$
J(x)=x^{*}(a) D x(a)+\int_{a}^{b} 2 \omega(t, x, u) d t
$$

is assumed elliptic relative to the inner product

where

$$
(x, y)=x^{*}(a) y(a)+\int_{a}^{b}\left(y^{*} x+v^{*} u\right) d t
$$

$$
x: x(t), \quad u(t), \quad y: y(t), \quad v(t)
$$

and

$$
2 \omega(t, x, u)=x^{*} P x+x^{*} Q u+u^{*} Q^{*} x+u^{*} R u .
$$

In the above let "*" denote the transpose of a matrix. The matrices $A, B, C$, and $D$ are respectively $n \times n, n \times q, n \times r$ and $n \times n$ constant real matrices where the rank of $C$ is $r \leqq n ; P(t)$ and $Q(t)$ are $n \times n$ and $n \times q$ Lebesgue square integrable matrices on $[a, b]$ with $P(t)=P^{*}(t)$; and $R(t)=R^{*}(t)$ is a $q \times q$ essentially bounded and Lebesgue integrable matrix on $[a, b]$ satisfying $R(t) \geqq \varepsilon I$ almost everywhere for some $\varepsilon>0$. The ellipticity of $J$ is a consequence of the fact that $R$ is positive definite in this sense.

For each $\lambda$ in $[a, b]$ let $\mathscr{C}(\lambda)$ be given by $\mathscr{C}(\lambda)=\{x$ in $\mathscr{C} \mid x(t)=0, u(t)=0$ a.e. on $\lambda \leqq t \leqq b\}$. Let $s(\lambda)$ and $n(\lambda)$ denote the signature and nullity of $J(x)$ on $\mathscr{C}(\lambda)$. We note that $\lambda_{1}<\lambda_{2}$ implies $\mathscr{C}\left(\lambda_{1}\right) \subset \mathscr{C}\left(\lambda_{2}\right)$ and that (3) holds with $\mathscr{C}$ and $\mathscr{C}(\lambda)$ replacing $\mathfrak{U}$ and $\mathfrak{U}(\lambda)$ respectively.

Intuitively the difference between the usual focal point phenomena and focal interval phenomena is the concept of abnormality. In the latter case a nonzero solution of Euler's equation (satisfying the transversality conditions) is allowed to equal zero on a subinterval of $[a, b]$. This is impossible in the former case. 
Mikami [9] has shown that if the matrices $A$ and $B$ are analytic in $[a, b]$ then, for such solutions, $x(t)=0$ on some proper subinterval $\left[a^{\prime}, b^{\prime}\right]$ of $[a, b]$ implies $x(t)=0$, $u(t)=0$ a.e. on $[a, b]$. Thus all focal intervals degenerate to focal points in this case.

As our final effort we will consider focal intervals in the setting of this example. Of special interest are the differential equations and boundary conditions associated with extremal solutions and their correspondence with the null vectors and focal intervals of §III. We note that the notion of focal intervals in an optimal control setting was suppressed in [9]. Hazard [5] defines focal intervals for the problem of Bolza in the calculus of variations setting, in terms of differential equations and "side" conditions.

An arc $x$ in $\mathfrak{B} \cap \mathscr{C}^{\mathrm{J}}$ is called a focal arc. From [9] we have: $x$ is a focal arc if and only if there exists an adjoint vector $p=\left(p^{1}, \ldots, p^{n}\right)$ and constant multipliers $v=\left(v^{1}, \ldots, v^{r}\right)$ such that the vector

$$
x p: x(t), \quad u(t), \quad p(t)
$$

satisfies the Euler-Lagrange equations

$$
x=A x+B u, \quad \dot{p}+A^{*} p=\omega_{x}, \quad B^{*} p=\omega_{u},
$$

the end conditions $C^{*} x(a)=0$, and the transversality condition $p(a)=D x(a)+C v$. In the above $p^{i}$ is absolutely continuous and $\dot{p}^{i}$ is Lebesgue square integrable on the interval $[a, b]$. Thus

THEOREM 21. If $x \neq 0$ is in $\mathscr{C}_{0}(\lambda)$ for $\lambda$ in $I=\left[\lambda^{\prime}, \lambda^{\prime \prime}\right]$, and not in $\mathscr{C}_{0}(\lambda)$ for $\lambda$ in an extension of $I$; there exists a focal arc $y$ which is identically zero on I and not identically zero on an extension of $I$, such that $x(t)=y(t)$ on $\left[a, \lambda^{\prime \prime}\right]$ and $x(t)=0$ on $\left[\lambda^{\prime}, b\right]$.

This follows for if $x$ in $\mathscr{C}_{0}\left(\lambda^{\prime \prime}\right)$, it coincides with a focal arc $y$ on the interval $\left[a, \lambda^{\prime \prime}\right]$. The arc $y$ has the properties of the theorem.

The converse is not true. For if $y$ is a focal arc described in Theorem 21 there may exist a focal arc $z$ which is zero on $\left[a, \lambda^{\prime \prime}\right]$ and satisfies $z(t)=y(t)$ on $\left[\lambda^{\prime \prime}, \bar{\lambda}\right]$ for $\lambda^{\prime \prime}<\bar{\lambda} \leqq b$. The focal arc $y_{1}=y-z$ "extends" the interval on which $x$ is a null vector. That is $x$ is in $\mathscr{C}_{0}(\lambda)$ for $\lambda$ in $\left[\lambda^{\prime}, \bar{\lambda}\right]$. This discussion partially motivates the following definition.

Let $a<\lambda^{\prime} \leqq \lambda^{\prime \prime} \leqq b$. A focal arc $y$ is called a maximum focal arc associated with an interval $I=\left[\lambda^{\prime}, \lambda^{\prime \prime}\right]$ if (i) $y$ is identically zero on $I$, (ii) $y$ is not identically zero on an extension of $I$, and (iii) there exists no focal arc $y_{1}$ having $y_{1}(t)=y(t)$ on an extension of $I$ to the left and $y_{1}(t)=0$ on an extension of $I$ to the right. By "extension" we mean an interval extension which includes $I$. This definition was used by Hazard [5] to define focal intervals.

The condition "(iv) there exists no focal arc $y_{2}$ having $y_{2}(t)=y(t)$ on an extension of $I$ to the right and $y_{2}(t)=0$ on an extension of $I$ to the left" is necessary to our definition. However it is redundant, for if $y_{2}$ satisfies (iv) the arc $y_{1}=y-y_{2}$ satisfies (iii) and conversely. 
The interval $I=\left[\lambda^{\prime}, \lambda^{\prime \prime}\right]$ will be called a maximum focal interval if there exists a maximum focal arc associated with $I$. The order of the maximum focal interval is the member $n$ of linearly independent maximal focal arcs $y_{1}, \ldots, y_{n}$ in a maximum set, every proper linear combination corresponding to $I$.

THEOREM 22. Let $x=x_{k l}$ in $\mathscr{C}_{0}(\lambda), \lambda^{\prime} \leqq \lambda \leqq \lambda^{\prime \prime}$, be a null vector associated with a focal interval $I=I_{i l l}=\left[\lambda^{\prime}, \lambda^{\prime \prime}\right]$ described in $\S \mathrm{III}$. Then there corresponds a unique maximal focal arc $y$ associated with I such that $x(t)=y(t)$ on $\left[a, \lambda^{\prime \prime}\right]$ and $x(t)=0$ on $\left[\lambda^{\prime}, b\right]$.

Let $x_{1}, \ldots, x_{m}$ be a maximum set of $m$ linearly independent arcs associated with a focal interval $I=I_{k l}$ described in $\S I I I$. By Theorem 21 there exists focal arcs $y_{1}, \ldots, y_{m}$ vanishing on $I$ and no extension of $I$ such that $x_{i}(t)=y_{i}(t)$ for $t$ in $\left[a, \lambda^{\prime \prime}\right]$; furthermore $x_{i}(t)=0$ on $\left[\lambda^{\prime}, b\right]$. If $\alpha_{j} y_{j}(t)=0(j=1, \ldots, m ; j$ summed $)$ on $a \leqq t \leqq b$ then $\alpha_{j} x_{j}(t)=0$ on $a \leqq t<b$ so that $\alpha_{j}=0$ for $j=1, \ldots, m$. Thus if $I$ is a maximal focal interval of order $n$ we have $n \geqq m$.

Conversely if $n>m$ there exists $m+1$ linearly independent maximal focal arcs $y_{1}, \ldots, y_{m+1}$ associated with $I$. Let $x_{i}(t)=y_{i}(t)$ on $\left[a, \lambda^{\prime \prime}\right]$ and $x_{i}(t)=0$ on $\left[\lambda^{\prime}, b\right]$ for $i=1, \ldots, m+1$. Let $\alpha_{1}, \ldots, \alpha_{m+1}$ be real and chosen such that $\alpha_{j} \alpha_{j} \neq 0$ and $\alpha_{j} x_{j}(t)=0$ on $[a, b]$ for $j=1, \ldots, m+1 ; j$ summed. Then $\alpha_{j} y_{j}(t)=0$ for $t$ in $\left[a, \lambda^{\prime \prime}\right]$ which contradicts the requirement that every proper linear combination vanish only on $I$.

COROLlaRY 23. If $\lambda$ in $[a, b]$ then $s(\lambda)$ is equal to the number of maximal focal intervals on the open interval $(a, \lambda)$.

This result follows immediately from Lemma 15.

\section{REFERENCES}

1. G. D. Birkhoff and M. R. Hestenes, Natural isoperimetric conditions in the calculus of variations, Duke Math. J. 1 (1935), 198-286.

2. R. F. Dennemeyer, Quadratic forms in Hilbert space and second order elliptic differential equations, Dissertation, University of California, Los Angeles, 1956.

3. J. Gregory, An approximation theory for elliptic quadratic forms on Hilbert spaces, Dissertation, University of California, Los Angeles, 1969.

4. - An approximation theory for elliptic quadratic forms on Hilbert spaces: Application to the eigenvalue problem for compact quadratic forms, Pacific J. Math. (to appear).

5. K. E. Hazard, Index theorems for the problem of Bolza in the calculus of variations, Contributions to the Calculus of Variations, 1938-1941, Univ. of Chicago Press, Chicago, Ill., 1942, pp. 293-356. MR 4, 47.

6. M. R. Hestenes, Applications of the theory of quadratic forms in Hilbert space to the calculus of variations, Pacific J. Math. 1 (1951), 525-582. MR 13, 759.

7. A. S. Householder, The dependence of a focal point upon curvature in the calculus of variations, Contributions to the Calculus of Variations, 1937, Univ. of Chicago Press, Chicago, Ill. 
8. G. C. Lopez, Quadratic variational problems involving higher order ordinary derivatives, Dissertation, University of California, Los Angeles, 1961.

9. E. Mikami, Quadratic optimal control problems, Dissertation, University of California, Los Angeles, 1968.

10. M. Morse, The calculus of variations in the large, Amer. Math. Soc. Colloq. Publ., vol. 18, Amer. Math. Soc., Providence, R. I., 1934.

11. W. T. Reid, An integro-differential boundary value problem, Amer. J. Math. 60 (1938), 257-292.

UNIVERSITY OF CALIFORNIA,

Los ANgeles, California 90024 